\title{
The MoEDAL Experiment at the LHC - a New Light on the High Energy Frontier
}

\author{
James L. Pinfold ${ }^{* \dagger}$ \\ University of Alberta E-mail: jpinfold@ualberta.ca
}

\begin{abstract}
MoEDAL is a pioneering experiment designed to search for highly ionising messengers of new physics such as magnetic monopoles or massive (pseudo-)stable charged particles, that are predicted to existing a plethora of models beyond the Standard Model. It started data taking at the LHC at a centre-of-mass energy of $13 \mathrm{TeV}$, in 2015. Its ground breaking physics program defines a number of scenarios that yield potentially revolutionary insights into such foundational questions as: are there extra dimensions or new symmetries; what is the mechanism for the generation of mass; does magnetic charge exist; and what is the nature of dark matter. MoEDAL's purpose is to meet such far-reaching challenges at the frontier of the field. We will present the first results from the MoEDAL detector on Magnetic Monopole production that are the world's best for Monopoles with multiple magnetic charge. In conclusion, plans to install a new MoEDAL subdetector designed to search for very long-lived neutral particles as well as mini-charged particles will be very briefly discussed.
\end{abstract}

The European Physical Society Conference on High Energy Physics

5-12 July

Venice, Italy

* Speaker.

${ }^{\dagger}$ For the MoEDAL Collaboration 


\section{Introduction}

The MoEDAL experiment, the newest LHC experiment, started data taking in 2015. MoEDAL is a pioneering experiment designed to search for highly ionizing avatars of new physics such as magnetic monopoles or massive (pseudo-)stable charged particles. It is largely deployed in the LHCb's VELO cavern at Point 8 (IP8) on the LHC ring. Its groundbreaking physics program defines over 30 scenarios that yield potentially revolutionary insights into such foundational questions as: are there extra dimensions or new symmetries; what is the mechanism for the generation of mass; does magnetic charge exist; what is the nature of dark matter; and, how did the big-bang develop. MoEDAL's purpose is to meet such far-reaching challenges at the frontier of the field.

The innovative MoEDAL detector employs unconventional methodologies tuned to the prospect of discovery physics. The largely passive MoEDAL detector has a dual nature. First, it acts like a giant camera, comprised of nuclear track detectors - analyzed offline by ultra fast scanning microscopes - sensitive only to new physics. Second, it is uniquely able to trap the particle messengers of physics beyond the Standard Model for further study. MoEDAL's radiation environment is monitored by a state-of-the-art real-time TimePix pixel detector array. MoEDAL is currently installing a prototype of the MAPP (MoEDAL Apparatus for very Penetrating Particles) sub-detector, in a tunnel in the vicinity of LHCb/MoEDAL at IP8. This new subdetector is designed to search for very long-lived highly penetrating particles such a mini-charged particles, long lived neutrals, etc.

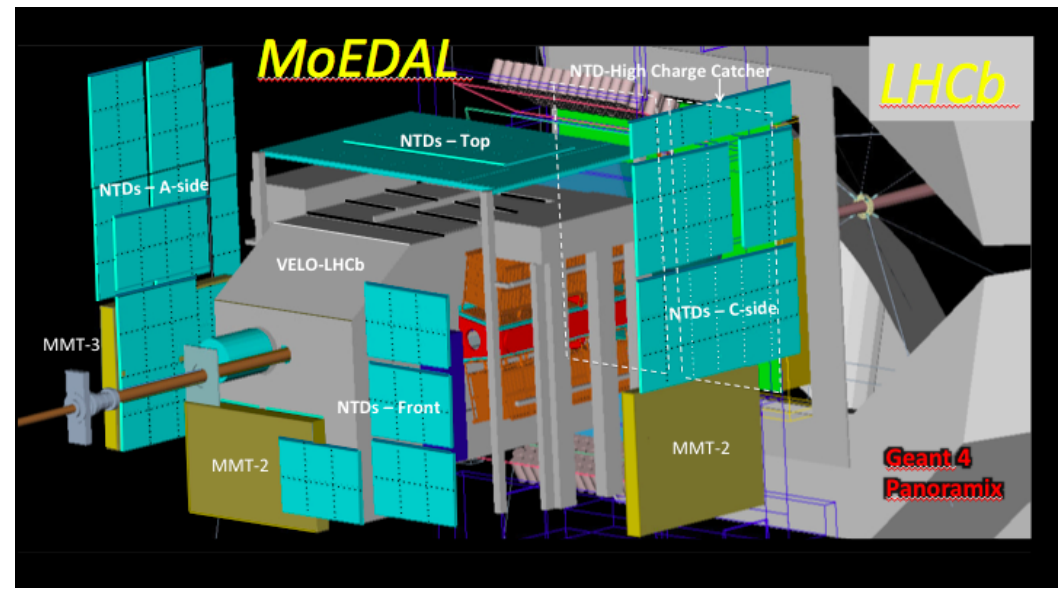

Figure 1: A GEANT-4 "Panoramix" depiction of the baseline MoEDAL detector.

\section{The MoEDAL Detector}

MoEDAL is an unconventional and largely passive LHC detector comprised of the largest array of plastic (CR39 and Makrofol) Nuclear Track Detector stacks ever deployed at an accelerator, surrounding the intersection region at Point 8 on the LHC ring. Another novel feature is the use of paramagnetic (aluminium) trapping volumes to capture both electrically and magnetically charged highly-ionizing particles predicted in new physics scenarios. It includes an array of TimePix pixel devices for monitoring highly-ionizing particle backgrounds. The main passive elements of the 
MoEDAL detector do not require a trigger system, electronic readout, or online computerized data acquisition. A GEANT-4 "Panoramix" depiction of the MoEDAL detector is given in Fig. 1.

MoEDAL's new planned MAPP subdetector is based on conventional scintillator detector technology readout by PMTs that takes advantage of a location protected by $30 \mathrm{~m}$ to $50 \mathrm{~m}$ or rock by Standard Model particles emanating from IP8. The baseline MoEDAL detector and its MAPP extension is designed to be largely complementary in its sensitivity to the main general-purpose LHC experiments ATLAS and CMS.

\section{The Physics Program}

The most important motivation for the MoEDAL experiment is to continue the quest for the magnetic monopole [1] to LHC energies. However, the experiment is also designed to search for massive, stable or long-lived, slow-moving particles [2] with single or multiple electric charges that arise in many scenarios of physics beyond the Standard Model (BSM) [1]. An overview of the over 30 physics topics that can be explored using the baseline experiment is shown in Fig. 2. The MAPP sub-detector will give access to a number of additional new physics scenarios involving

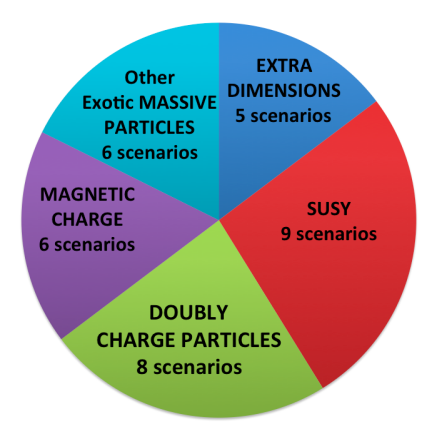

Figure 2: A pie chart describing the topics covered by MoEDAL's physics program

fractionally charged particles and long lived neutral particles, etc. The MoEDAL physics reach is largely complementary to the that of the large multi-purpose LHC detectors ATLAS and CMS.

\section{MoEDAL Results and Current Status}

MoEDAL first published physics analysis reported a search for magnetic monopoles using a $160 \mathrm{~kg}$ prototype MoEDAL trapping detector exposed to $8 \mathrm{TeV}$ proton-proton collisions [3] at the LHC, for an integrated luminosity of $0.75 \mathrm{fb}^{-1}$. In this case monopoles were sought by passing the trapping volumes through a superconducting magnetometer (SQUID) in order to detect the presence of induced persistent currents caused by the passage of a monopole through the SQUID. No magnetic charge exceeding $0.5 \mathrm{~g}_{D}$ (where $\mathrm{g}_{D}$ is the Dirac magnetic charge) is measured in any of the exposed samples, allowing limits to be placed on monopole production in the mass range 100 $\mathrm{GeV} \leq m \leq 3500 \mathrm{GeV}$. Model-independent cross-section limits are presented in fiducial regions of monopole energy and direction for $1 \mathrm{~g}_{D} \leq|\mathrm{g}| \leq 6 \mathrm{~g}_{D}$, and model-dependent cross-section limits are obtained for Drell-Yan pair production of spin- $1 / 2$ and spin- 0 monopoles for $1 \mathrm{~g}_{D} \leq|g| \leq 4 \mathrm{~g}_{D}$. 
Under the assumption of Drell-Yan cross sections, mass limits are derived for $|\mathrm{g}|=2 \mathrm{~g}_{D}$ and $|\mathrm{g}|=$ $3 \mathrm{~g}_{D}$ for the first time at the LHC, surpassing the results from previous collider experiments.

A second MoEDAL analysis presented the search for magnetic monopole production in run-2 $13 \mathrm{TeV}$ proton-proton collisions [4] using the trapping technique, extending a previous publication with $8 \mathrm{TeV}$ data during LHC run-1. A total of $222 \mathrm{~kg}$ of MoEDAL trapping detector samples was exposed in the forward region and then monitored for the presence of magnetic monopoles in a data sample corresponding to a luminosity of $0.371 \pm 0.004 \mathrm{fb}^{-1}$ obtained during the 2015 exposure to $13 \mathrm{TeV}$ pp collisions. The results of both analyses are summarized in Fig. 3. Monopole-pair direct production cross-section limits in the range $200 \rightarrow 10000 \mathrm{fb}$ were set for magnetic charges up to $5 \mathrm{~g}_{D}$ and masses up to $6 \mathrm{TeV}$. In a DY model with spin- $1 / 2$ monopoles, this translates into monopole mass limits exceeding $1 \mathrm{TeV}$ - the strongest to date at a collider experiment - for charges ranging from two to four times the Dirac charge. These were the first monopole search results published at $13 \mathrm{TeV}$ LHC centre-of-mass energy.

\begin{tabular}{|l|c|c|c|c|}
\hline mass limits [GeV] & $1 g_{\mathrm{D}}$ & $2 g_{\mathrm{D}}$ & $3 g_{\mathrm{D}}$ & $4 g_{\mathrm{D}}$ \\
\hline $\begin{array}{l}\text { MoEDAL 13 TeV } \\
\text { (this result) }\end{array}$ & & & & \\
DY spin-1/2 & 890 & 1250 & 1260 & 1100 \\
DY spin-0 & 460 & 760 & 800 & 650 \\
\hline MoEDAL 8 TeV & & & & \\
DY spin-1/2 & 700 & 920 & 840 & - \\
DY spin-0 & 420 & 600 & 560 & - \\
\hline ATLAS 8 TeV & & & & \\
DY spin-1/2 & 1340 & - & - & - \\
DY spin-0 & 1050 & - & - & - \\
\hline
\end{tabular}

Figure 3: Monopole lower mass limits (95\% confidence level) in models of spin- $1 / 2$ and spin- 0 DY pair production in LHC pp collisions for monopole charges $\mid \mathrm{gl}$ up to $4 \mathrm{~g}_{D}$. These limits are based upon cross sections computed at leading order. These cross sections are only indicative since the monopole coupling to the photon is too large to allow for perturbative calculations. Previous results obtained in $8 \mathrm{TeV}$ collisions are from Ref. [3] (MoEDAL prototype trapping detector) and Ref. [5] (ATLAS).

Currently, we have several analyses in the pipeline where the full functionality of the MoEDAL detector is employed to search for anomalously ionizing new particles in both proton-proton and heavy ion collisions.

\section{References}

[1] For example: B. Acharya et al., Int. J. Mod. Phys. A29, 1430050 (2014).

[2] For example: M. Fairbairn, A. C. Kraan, D. A. Milstead, T. Sjostrand, P. Z. Skands and T. Sloan, Phys. Rept. 438, 1 (2007).

[3] B. Acharya et al., JHEP 1608, 067 (2016).

[4] B. Acharya et al., Phys. Rev. Lett. 118,no. 6, 061801 (2017).

[5] G. Aad et al, ATLAS Collaboration, Phys. Rev. D93, 052009 (2016). 